\title{
Virtual-detector synthetic aperture focusing technique with application in in vivo photoacoustic microscopy
}

Meng-Lin Li, Hao F. Zhang, Konstantin Maslov, George Stoica, Lihong V. Wang

Meng-Lin Li, Hao F. Zhang, Konstantin Maslov, George Stoica, Lihong V. Wang, "Virtual-detector synthetic aperture focusing technique with application in in vivo photoacoustic microscopy," Proc. SPIE 6086, Photons Plus Ultrasound: Imaging and Sensing 2006: The Seventh Conference on Biomedical Thermoacoustics, Optoacoustics, and Acousto-optics, 60861F (6 March 2006); doi: 10.1117/12.645115 


\title{
Virtual-detector synthetic aperture focusing technique with application in in-vivo photoacoustic microscopy
}

\author{
Meng-Lin Li ${ }^{\mathrm{a}}$, Hao. F. Zhang ${ }^{\mathrm{a}}$, Konstantin Maslov ${ }^{\mathrm{a}}$, George Stoica ${ }^{\mathrm{b}}$, and Lihong V. Wang ${ }^{\mathrm{a}}$ \\ ${ }^{a}$ Optical Imaging Laboratory, Department of Biomedical Engineering, Texas A\&M University, \\ College Station, TX 77843-3120 \\ ${ }^{b}$ Department of Veterinary Pathobiology, Texas A\&M University, College Station, TX 77843-5547 \\ Corresponding author: Email: lwang@tamu.edu; TEL: 979-847-9040; FAX: 979-845-4450
}

\begin{abstract}
In this study, we introduce a synthetic aperture focusing technique which employs a virtual detector concept, combined with coherence weighting, to extend the depth of focus for an in-vivo photoacoustic microscopy system. This technique treats the transducer's focal point as a virtual point detector of photoacoustic signals, delays adjacent scan lines relative to the virtual detector, and then sums the delayed signals to achieve focusing in the out-of-focus region. In addition, a coherence factor among the delayed signals for each synthesized imaging point is used as a weighting factor to further improve the focusing quality. Images of an Intralipid phantom containing a carbon fiber show how this technique improves the $-6 \mathrm{~dB}$ lateral resolution from $49-379 \mu \mathrm{m}$ to $46-53 \mu \mathrm{m}$ and increases the SNR by $0-29 \mathrm{~dB}$, depending on the distance from the ultrasonic focal point. In vivo experiments show that this technique also provides a clearer tumorassociated angiogenesis in the mouse's scalp. The extended depth of focus for the photoacoustic microscopy system enables 3D reconstruction of the vascular network for the study of tumor angiogenesis.
\end{abstract}

Keywords: Photoacoustic microscopy, virtual detector, synthetic aperture focusing technique, coherence weighting, depth of focus

\section{INTRODUCTION}

Photoacoustic imaging has been applied to measuring the optical properties of biological tissue noninvasively. Photoacoustic signals can be induced with illumination from a pulsed laser. When biological tissues absorb the pulsed laser energy, due to thermoelastic expansion, ultrasonic signals are generated, which can be detected and then utilized to reconstruct the optical absorption distribution in the tissues. Photoacoustic imaging combines the merits of both optics and ultrasound. Therefore, it provides good optical contrast with high acoustic resolution. It has been shown to be a promising tool for vasculature structural imaging [1]-[3], epidermal melanin measurement [4][5], breast tumor detection [5], and oxygenation monitoring in blood vessels [7]. Recently, an in vivo backward-mode confocal photoacoustic microscope (PAM) with dark-field illumination was developed by Maslov et al. to image microvascular networks in the skin [8]. This system employed a high-frequency, wide-band, large numerical-aperture spherically focused transducer, which is coaxial and confocal with the optical illumination, to achieve high image resolution and high sensitivity. This system can provide high lateral resolution, $45 \mu \mathrm{m}$ at the focal point, and high axial resolution, $\sim 15 \mu \mathrm{m}$, and it is capable of imaging optical absorption contrasts as deeply as $3 \mathrm{~mm}$ into biological tissue. This PAM system shows great potential for applications in dermatology and related cancer research.

However, the drawback of using such a large numerical-aperture acoustic lens is the limited depth of focus, which causes the image quality to deteriorate significantly in the out-of-focus region. The idea of using a synthetic aperture focusing technique (SAFT) to improve the resolution and the signal-to-noise ratio (SNR) in photoacoustic imaging has been presented previously [9]. However, a point detector (e.g., a needle hydrophone) is needed for such conventional SAFT. Here, we introduce a virtual-point-detector-based synthetic aperture focusing technique to extend the depth of focus for PAM using a large numerical-aperture transducer. In addition, coherence among the delayed signals for each synthesized imaging point in the SAFT is used as a weighting factor to further improve the SAFT image quality. 


\section{VIRTUAL-DETECTOR-BASED SYNTHETIC APERTURE FOCUSING TECHNIQUE}

As illustrated in figure 1(a), in virtual-detector-based SAFT, the focal point of the transducer is treated as a virtual point detector. When biological tissues absorb pulsed laser energy, photoacoustic waves generated within a certain solid angle above and below the virtual detector (i.e., focal point) are assumed to propagate toward, and then be detected by, the virtual detector. If a linear scan is performed, the photoacoustic radiation pattern from the virtual detector at the current position will overlap with that produced at adjacent positions. The synthetic aperture focusing can be done in the overlapped region of these photoacoustic radiation patterns below and above the virtual detectors.

Figure 1(b) shows the focusing geometry of the virtual-detector based SAFT. The scan line $i$ is at a distance of $x_{i}$ away from the synthesized beam axis. The photoacoustic radiation pattern of the scan line $i$ covers the desired synthetic focal point $p$. Therefore, the photoacoustic signals from scan line $i$ can be added to the synthesized beam constructively by applying an appropriate time delay. Based on the virtual detector concept, the time delay applied to the received signal of scan line $i$ can be expressed as:

$\Delta t_{i}=\operatorname{sgn}\left(z-z_{f}\right) \cdot \frac{r_{i}{ }^{\prime}-r}{c}$,

where $z_{\mathrm{f}}$ is the transducer's focal length; $z$ is the depth of the synthetic focal point $p$ from the transducer surface; $r_{i}{ }^{\prime}$ is the distance from the virtual detector $f_{i}$ of scan line $i$ to the synthetic focal point; $r$ is the axial distance from the transducer's focal point to the synthetic focal depth; $c$ is the acoustic velocity; and $\operatorname{sgn}(\cdot)$ is the signum function. Note that, if the synthetic focal point is above the focal point of the transducer, the time delay applied is negative. The virtual-detector based SAFT is implemented by applying appropriate delays, relative to the virtual point detectors, to the adjacent scan lines according to (1) and then summing the delayed signals [10]:

$S_{S A F T}(t)=\sum_{i} S\left(i, t-\Delta t_{i}\right)$,

where $S(i, t)$ is the received signal at scan line $i$. Note that the maximum number of scan lines included in the sum of $(2)$ is determined by the angular extent of the photoacoustic radiation pattern, which is dominated by the parameters of the ultrasonic transducer because the light distribution is much broader due to the strong scattering nature of light in biological tissue. Moreover, SAFT can also increase the SNR in the out-of-focus region.

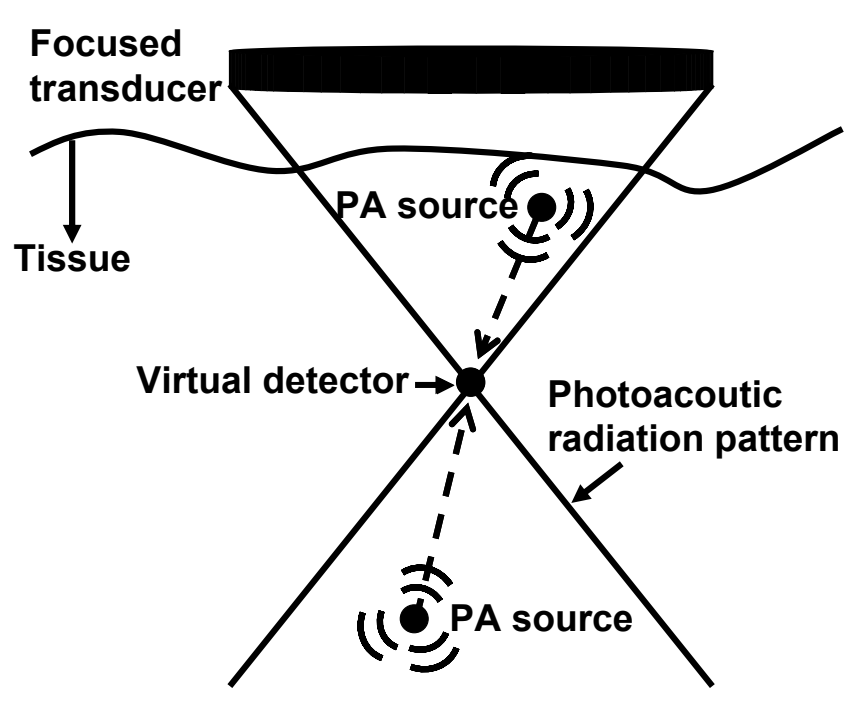

(a)

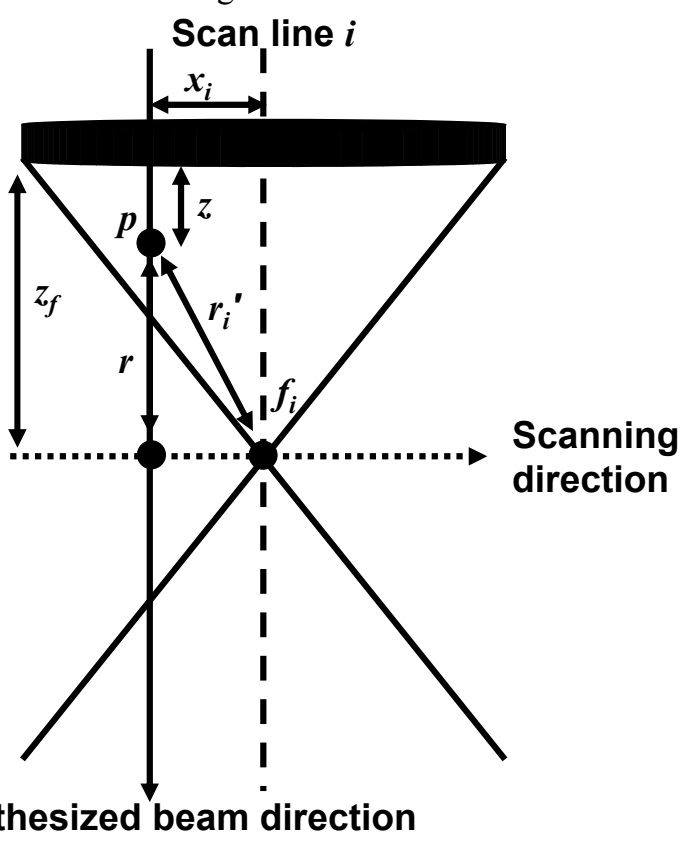

(b)

Fig. 1 (a) Illustration of the virtual detector concept (b) Focusing geometry of the virtual-detector based SAFT 


\section{COHERENCE-FACTOR WEIGHTING}

In addition to virtual-detector-based SAFT, a coherence factor (CF) among the delayed signals for each SAFT imaging point is employed as a weighting factor to further improve the focusing quality. The definition of CF is [9]:

$$
\mathrm{CF}(t)=\frac{\left|\sum_{i} S\left(i, t-\Delta t_{i}\right)\right|^{2}}{N \cdot \sum_{i}\left|S\left(i, t-\Delta t_{i}\right)\right|^{2}},
$$

where $N$ is the number of scan lines included in the SAFT sum of (2). CF is a quantitative measure of coherence among the delayed signals (i.e., indicator of the degree of in-phase addition among the delayed signals) and ranges from 0 to 1. The idea of the CF weighting technique is illustrated in figure 2. In figure 2, the horizontal axis is the lateral position, and the vertical axis denotes the depth. The leftmost panel is the simulated point spread function (PSF) of the SAFT. The middle panel is the corresponding CF map. According to the definition of CF, due to the constructive summation of the delayed signals (i.e., highly in phase), the CF at the main lobe of the PSF is high (close to 1). However, the CFs at the side lobes are low because of the destructive summation of the delayed signals. Based on this property, as shown in the rightmost panel, the side lobes in the SAFT are suppressed after being multiplied by the corresponding CF map on a point-by-point basis, and hence the beam quality in the SAFT is improved. In addition, CF weighting can reduce the elevated side lobes in the SAFT caused by sound velocity inhomogeneities in the biological tissue [11]. CF weighting can also suppress background noise since the measurement noise is generally out-of-phase.

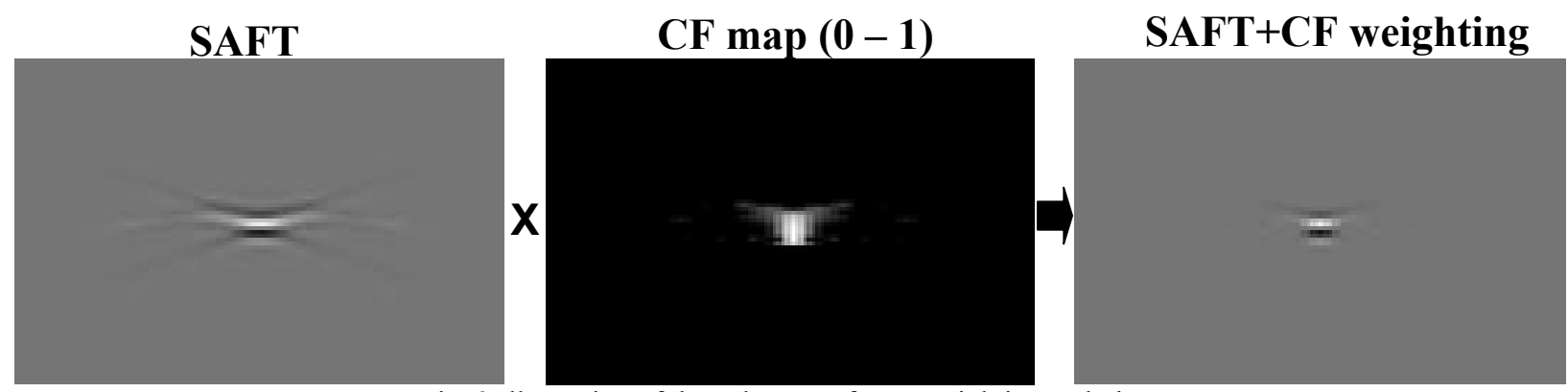

Fig. 2 Illustration of the coherence-factor weighting technique

\section{EXPERIMENTAL RESULTS}

In this study, the in vivo dark-field backward-mode PAM invented by Maslov et al. was used. Figure 3 shows the block diagram of this dark-field confocal PAM system. A tunable dye laser (ND6000, Continuum), pumped by an Nd:YAG laser (Brilliant B, Bigsky), was employed to provide laser pulses with a FWHM of $6.5 \mathrm{~ns}$, a wavelength of 584 $\mathrm{nm}$, and a pulse repetition rate of $10 \mathrm{~Hz}$. The laser energy was delivered by a $0.6-\mathrm{mm}$ diameter optical fiber whose output was coaxially positioned on a three-dimensional precision translation stage translating an ultrasonic transducer (V214-BC-RM, Panametrics) with a 50-MHz center frequency, a 70\% nominal bandwidth, and an in-house-constructed acoustic lens of 0.44 numerical-aperture. The laser induced photoacoustic signals were amplified by a broadband amplifier (ZFL-500N, Mini-Circuits) and then were digitized at a $250 \mathrm{MHz}$ sampling rate by a digital oscilloscope (TDS 5034B, Tektronics), which is triggered by the Nd:YAG laser and used to synchronize the motor controller. The incident energy density on the sample surface was controlled to $<1 \mathrm{~mJ} / \mathrm{cm}^{2}$. In the SAFT, each scan line was first interpolated by a factor of eight to increase the delay accuracy, and the acoustic velocity was assumed to be $1.5 \mathrm{~mm} / \mu \mathrm{s}$. Note that the PA images shown here were taken without any signal averaging to reduce the data acquisition time. 


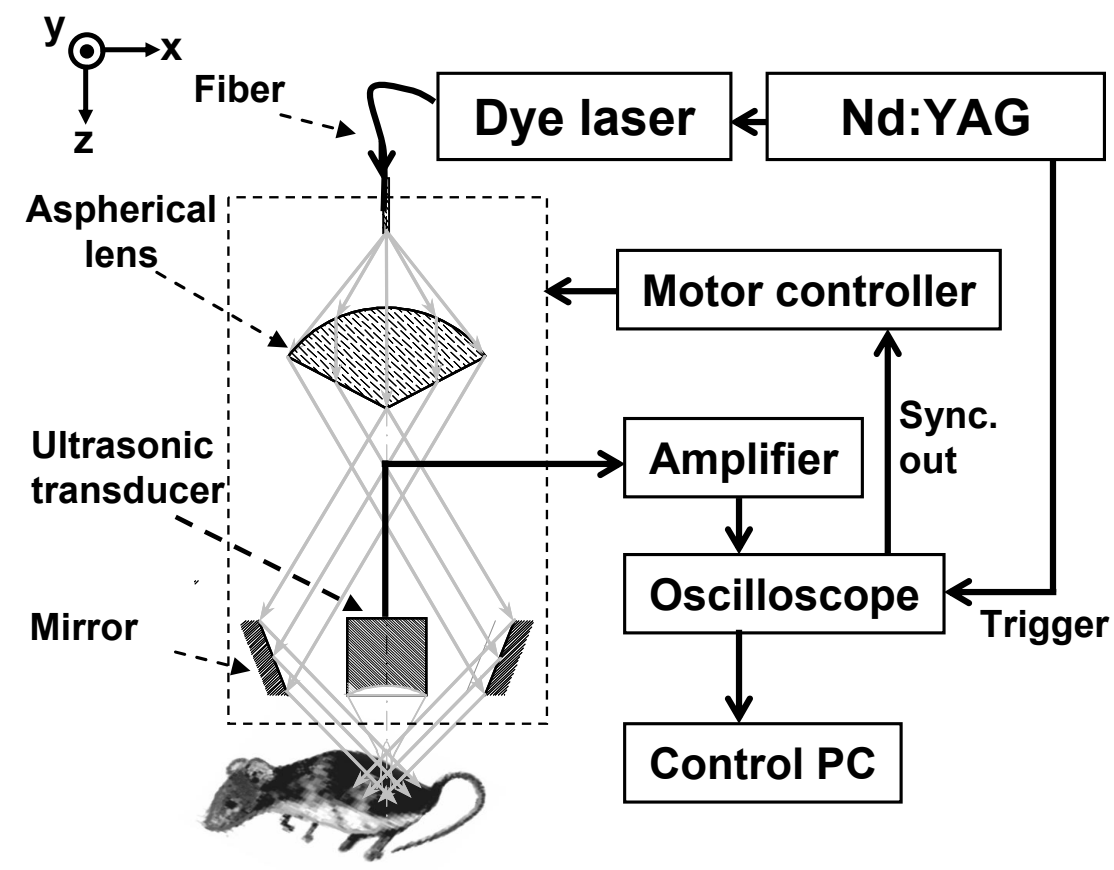

Fig. 3 Block diagram of the dark-field confocal photoacoustic microscope

4. 1 Intralipid phantom of a carbon fiber

(a) Original

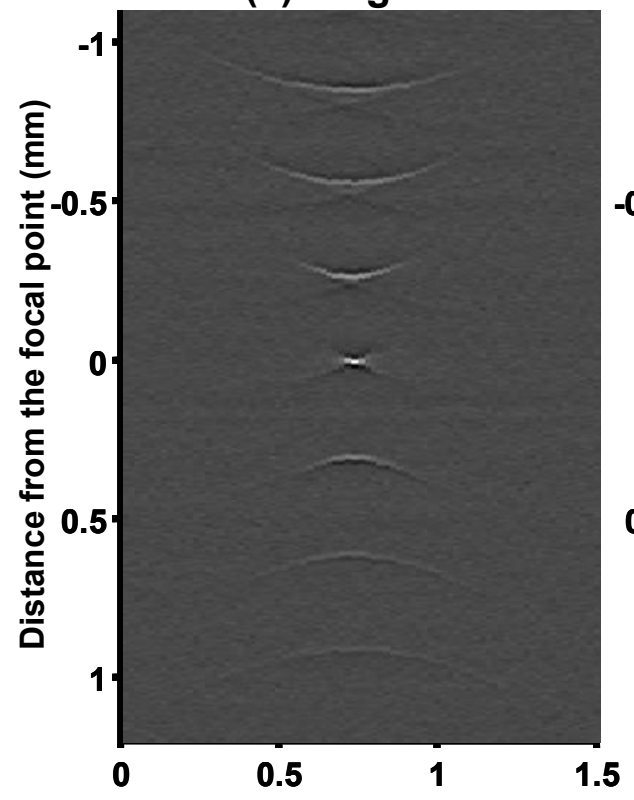

(b) SAFT+CF weighted

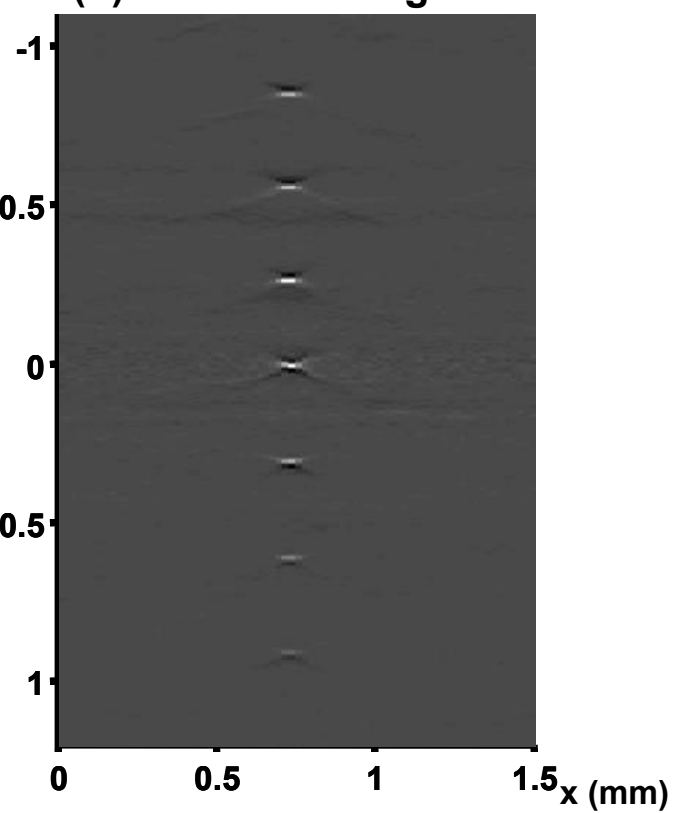

Fig. 4 (a) Original and (b) SAFT + CF weighted B-scan images of an Intralipid phantom containing a carbon fiber, which was scanned at different depths. The horizontal axis is the lateral scanning position, and the vertical axis is the distance from the focal point along the depth direction where a positive value means the depth is below the focal point and a negative value means the depth is shallower than the focal point. 
The performance of the virtual-detector based SAFT + CF weighting was evaluated using the PSF of the PAM. The PSF was measured by a cross-sectional imaging of a carbon-fiber phantom. This phantom was composed of a 6- $\mathrm{m}$ carbon fiber immersed in a diluted Intralipid solution (Clintec Nutrition Company, Dearfield, Illinois). This fiber was imaged at various depths, and images at different depths were montaged into one image, as shown in figure 4 . The scanning step size was $20 \mu \mathrm{m}$. Note that the maximum number of scan lines for the beam synthesis was limited, in this case, to 40. This limitation reduced the implementation complexity of the virtual-detector based SAFT. Figure 4 shows (a) the original B-scan image and (b) the SAFT + CF weighted image. The horizontal axis is the lateral position. The vertical axis is the axial distance from the imaging point to the focal point of the transducer in which a negative value means the depth is shallower than the focal point and a positive one indicates the imaging depth is deeper than the focal point. The two images are shown in the same linear gray scale. The brightness represents the laser-induced photoacoustic pressure detected by the transducer. In figure 4(a), due to the limited depth of focus of the transducer, the farther the carbon fiber is away from the focal point, the wider the lateral extent, which indicates poorer lateral resolution. Figure 4(b) shows that the SAFT + CF weighting significantly improves the lateral resolution.

The $-6-\mathrm{dB}$ lateral and axial resolutions with, and without the SAFT $+\mathrm{CF}$ weighting, at all of the imaging depths in figure 4 are estimated and shown in figure 5. In figure 5, the lateral axis is the axial distance from the imaging point to the focal point of the transducer. Figure 5(a) shows that the SAFT $+\mathrm{CF}$ weighting can provide depth independent lateral resolution. The proposed technique reduces the -6-dB width of the imaged carbon fiber from 49-379 $\mu \mathrm{m}$ to $46-$ $53 \mu \mathrm{m}$, depending on the distance from the ultrasonic focal point. As shown in figure 5(b), the SAFT $+C F$ weighting does not affect the axial resolution (the estimated $-6-\mathrm{dB}$ axial resolution is about $11 \mu \mathrm{m}$ ). In addition, the improved SNR from the SAFT + CF weighting is also estimated and shown in figure 6. Here, the SNR is defined as the ratio of the maximal intensity at the carbon fiber position to the average noise intensity. As shown in figure 6 , the SAFT $+\mathrm{CF}$ weighting can improve the SNR by up to $29 \mathrm{~dB}$. The farther the imaging depth is away from the focal point, the higher the improved SNR because more scan lines are included in the SAFT sum. By contrast, the SNR is not improved at the focal point because no SAFT summing is performed.

(a) -6 dB lateral resolution

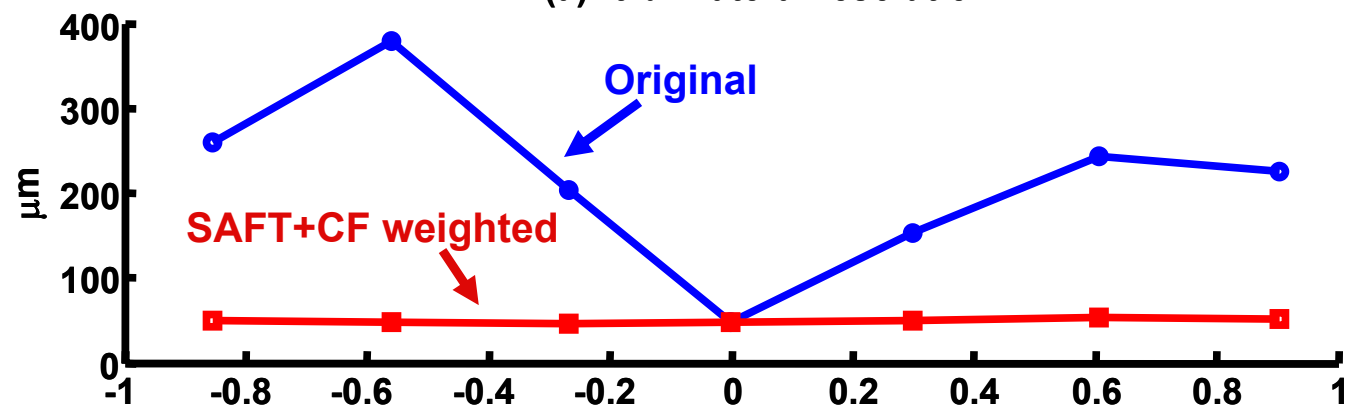

(b) $-6 \mathrm{~dB}$ axial resolution

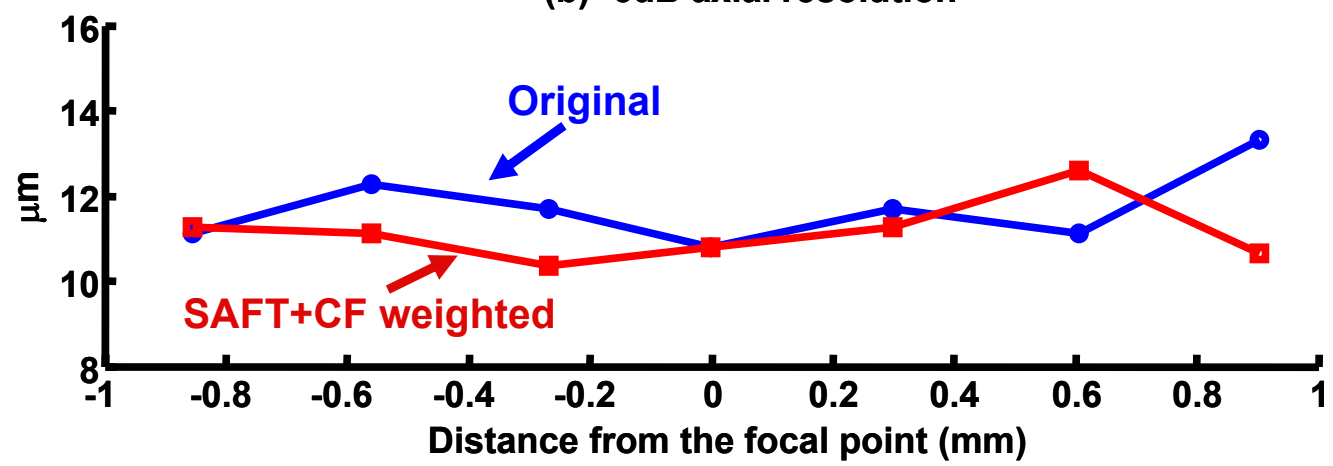

Fig. 5 The $-6 \mathrm{~dB}$ lateral and axial resolution with and without SAFT+CF weighting. (a): $-6 \mathrm{~dB}$ lateral resolution; (b) $-6 \mathrm{~dB}$ axial resolution. Lines with circles: original; lines with squares: $\mathrm{SAFT}+\mathrm{CF}$ weighted. 


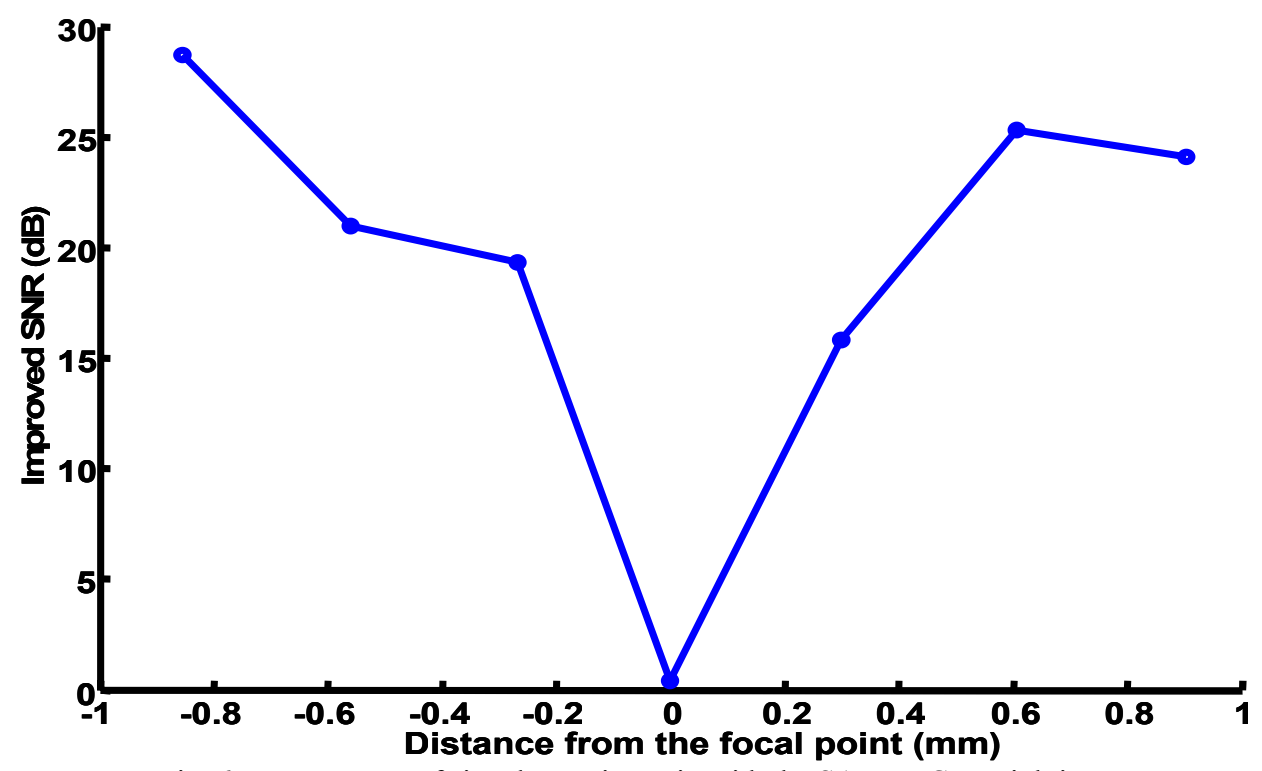

Fig. 6 Improvement of signal-to-noise ratio with the SAFT + CF weighting.

\section{2 In vivo imaging of mice}
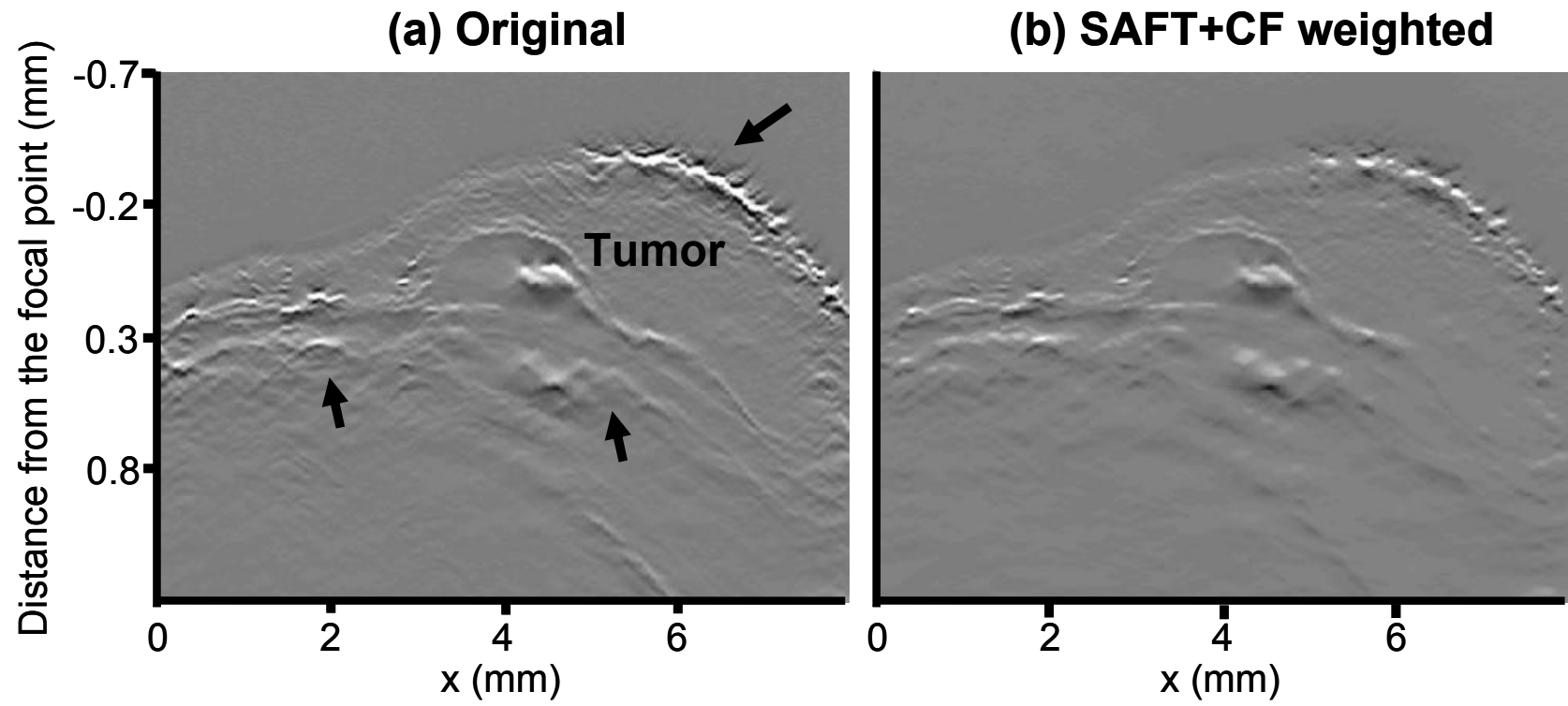

Fig. 7 In vivo B-scan images of the BALB/C mouse's scalp with tumor. (a): Original; (b) SAFT+CF weighted.

In vivo experiments on mice were also conducted to further evaluate the efficacy of the proposed technique. The scalps of BALB/C mice ( $20 \mathrm{~g})$ with CT26 tumors were imaged. Anesthesia was administrated by intramuscular injection of ketamine hydrochloride $(44 \mathrm{mg} / \mathrm{kg})$, xylazine hydrochloride $(2.5 \mathrm{mg} / \mathrm{kg})$, acepromazine maleate $(0.75$ $\mathrm{mg} / \mathrm{kg})$, and atropine $(0.025 \mathrm{mg} / \mathrm{kg})$. Before imaging, the hair on the mouse head was removed gently using commercial hair remover lotion. During the data acquisition, the mouse was provided pure oxygen for breathing and the arterial blood oxygenation level and heart rate were monitored by a pulse oximeter ( $8600 \mathrm{~V}$, NONIN). After the experiments, the mice recovered normally without any noticeable health problems. All experimental animal procedures were carried out in compliance with the guidelines of the U.S. National Institute of Health. The laboratory animal protocol for this work was approved by the University Laboratory Animal Care Committee of Texas A\&M University. 
(a) Original

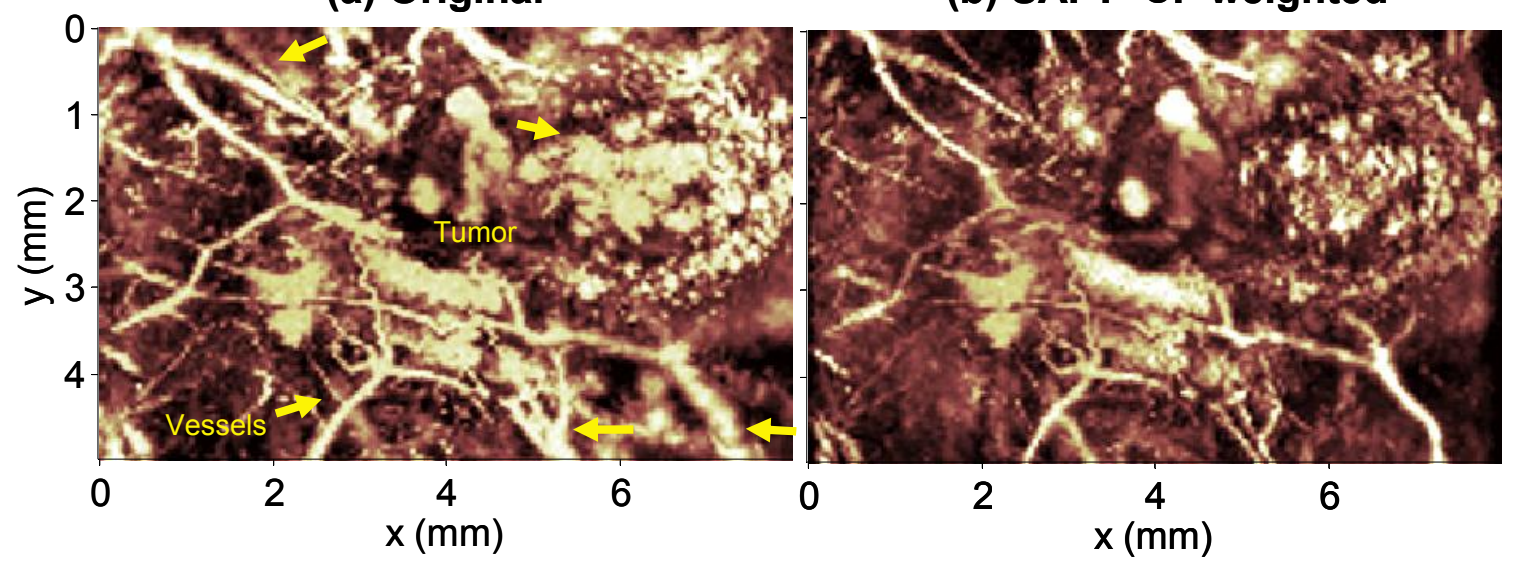

Fig. 8 In vivo projected C-scan images of the BALB/C mouse's scalp with tumor. (a): Original; (b) SAFT+CF weighted.

Figures 7 shows the in vivo original (a) and the SAFT $+\mathrm{CF}$ weighted (b) B-scan images of a mouse's scalp with a tumor. A scanning step size of $50 \mu \mathrm{m}$ was used to reduce the scanning time and to enlarge the imaging area. To reduce motion artifacts, the maximum number of scan lines included in the SAFT sum of (2) was limited to 10. The two images are displayed in the same linear gray scale. The vertical axis is the axial distance from the focal point of the transducer, and the horizontal is the lateral scanning position. Figure 7(b) exhibits better definition of the vessel positions than the original image 7(a) over the entire depth of the imaging field (e.g., the positions indicated by the arrows); and thus it shows clearer angiogenesis of the tumor. Figure 8(a) (original) and (b) (SAFT $+\mathrm{CF}$ weighted) are in vivo projected Cscan images of a mouse's scalp with the tumor displaying tumor associated angiogenesis. In figure 8 , the maximum of each scan line along the depth direction versus the two dimensional transducer scanning position is plotted. Figure $8(\mathrm{~b})$ provides a clearer vascular distribution than figure 8(a) as a result of the improved lateral resolution and SNR, as shown in figures 4,5 , and 6 . Note that here only one dimensional SAFT + CF weighting was performed for each B-scan along the $\mathrm{x}$ direction to reduce motion artifacts; hence only the lateral resolution in the $\mathrm{x}$ direction is improved.

\section{SUMMARY}

In summary, we introduced a virtual-detector-based synthetic aperture focusing technique, combined with coherence weighting, to extend the depth of focus of PAM with a large numerical-aperture transducer. The virtualdetector focusing technique significantly improves the degraded lateral resolution in the out-of-focus region of the PAM system when employing a large numerical-aperture ultrasonic transducer. The phantom and in vivo experimental results demonstrate the efficacy of this technique: depth independent lateral resolution is achieved; the SNR is also improved and, thus, clearer vascular distribution is obtained. In addition, if a higher pulse-rate laser is used to increase the scanning speed and thus reduce motion artifacts, two dimensional SAFT can be applied to obtain even better image quality. The extended depth of focus for the PAM system enables 3D reconstruction of the vascular network to extend research related to tumor angiogenesis.

\section{ACKNOWLEDGEMENTS}

We are grateful to Gina Lungu, Sergio Similache, and Ovidiu Cracium for assistance with cell culture and veterinary procedures. We also gratefully acknowledge funding from the National Institutes of Health, R01 EB000712 and R01 NS46214(BRP). L. V. Wang's e-mail address is lwang@oilab.tamu.edu. 


\section{REFERENCES}

1. X. Wang, Y. Pang, G. Ku, X. Xie, G. Stoica, and L. V. Wang, "Noninvasive laser-induced photoacoustic tomography for structural and functional in vivo imaging of the brain," Nat. Biotechnol. 21, pp. 803-806, 2003.

2. G. Ku, X. Wang, X. Xie, G. Stoica, and L. V. Wang, "Imaging of tumor angiogenesis in rat rains in vivo by photoacoustic tomography," Appl. Opt. 41, pp. 770-775,2005.

3. J. J. Niederhauser, M. Jaeger, R. Lemor, P. Weber, and M. Frenz, "Combined ultrasound and optoacoustic system for real-time high-contrast vascular imaging in vivo," IEEE Trans. Med. Imag. 24, pp. 436-440, 2005.

4. E. V. Savateeva, A. A. Karabutov, B. Bell, R. Johnigan, M. Motamedi, and A. A. Oraevsky, "Non-invasive detection and staging of oral cancer in vivo with confocal optoacoustic tomography," Proc. SPIE 3597, pp. 55-66, 2000.

5. J. A. Viator, L. O. Svaasand, G. Aguilar, B. Choi, and J. S. Nelson, "Photoacoustic measurement of epidermal melanin," Proc. SPIE 4960, pp. 14-20, 2003.

6. R. O. Esenaliev, A. A. Karabutov, and A. A. Oraevsky, "Sensitivity of laser opto-acoustic imaging in detection of small deeply embedded tumors," IEEE J. Sel. Top. Quantum Electron. 5, pp. 981- 988, 1999.

7. R. O. Esenaliev, I. V. Larina, K. V. Larin, D. J. Deyo, M. Motamedi, and D. S. Prough, "Optoacoustic technique for noninvasive monitoring of blood oxygenation: a feasibility study," Appl. Opt. 41, pp. 4722-4731, 2002.

8. K. Maslov, G. Stoica, and L. V. Wang, "In vivo dark-field reflection-mode photoacoustic microscopy," Opt. Lett. 30, pp. 625-627, 2005.

9. C.-K. Liao, M.-L. Li, and P.-C. Li, "Optoacoustic imaging with synthetic aperture focusing and coherence weighting," Opt. Lett. 29, pp. 2506-2508, 2004.

10. M.-L. Li, W. J. Guan, and P.-C. Li, "Improved synthetic aperture focusing technique with application in highfrequency ultrasound imaging," IEEE Trans. Ultrason., Ferroelect., Freq. Contr. 51, pp. 63- 70, 2004.

11. P.-C. Li and M.-L. Li, "Adaptive imaging using the generalized coherence factor," IEEE Trans. Ultrason., Ferroelect., Freq. Contr. 50, pp. 128-141, 2003. 